\title{
Valutazione clinica e metabolica dell'impiego dell'insulina Lispro in pazienti con diabete mellito scompensato
}

Antonio Uneddu*, Tito Antonio Paolini*

\begin{abstract}
The aim of the present work is to report preliminary results about the administration of an insulin analogue, LisPro, to treat diabetic chetoacidosis. This new procedure, in respect to insulin continuous infusion, allow significant cost reduction in terms of medical and nurses time dedicated to the patient and hospitalization length. In our experience, LisPro treatment compared to the standard of care, has allowed hospital time reduction of 2,1 days per patient. This can be translated in cost saving of about 700,00 euro per case. These preliminary data, replicating the results coming from published international experiences, have to be confirmed in our country through the implementation of more representative studies
\end{abstract}

Keywords: diabetic chetoacidosis, LisPro, insulin continuous infusion Farmeconomia e percorsi terapeutici 2005; 6 (4): 397-400

\section{INTRODUZIONE}

La chetoacidosi diabetica è la più comune emergenza iperglicemica nei pazienti con diabete. Rappresenta la principale causa di morte nei bambini con diabete di tipo 1 [1,2], e causa una quota significativa (pari a circa il 4\%-9\%), delle dimissioni relative a pazienti diabetici di tipo 1 o tipo $2[1,3]$. I cardini del trattamento della chetoacidosi sono tradizionalmente costituiti dalla somministrazione di insulina in infusione endovenosa continua o dalla frequente somministrazione di iniezioni sottocutanee o intramuscolari $[3,4,5]$. Sebbene numerosi studi controllati abbiano evidenziato che una terapia insulinica a basse dosi è efficace nella chetoacidosi diabetica indipendentemente dalla via di somministrazione [4,6-8], la strategia ideale rimane tuttora materia di discussione.

Queste procedure tuttavia richiedono un monitoraggio continuo e quindi un impiego intensivo del personale medico e infermieristico, con costi rilevanti per la struttura. Due recenti esperienze internazionali $[9,10]$ hanno confrontato questo approccio classico, condotto in reparti intensivi, con quello che prevede la somministrazione dell'analogo dell'insulina Lispro in un regime non inten- sivo di assistenza. Lo studio di Umpierrez et al [10] ha confrontato, prospetticamente, 20 pazienti in stato di chetoacidosi trattati con insulina Lispro subcutanea in reparti sub-intensivi con altrettanti pazienti trattati intensivamente con insulina normale. Anche la ricerca di Della Manna et al [9] ha utilizzato un disegno prospettico in cui sono stati randomizzati a ricevere insulina Lispro o insulina normale, in regime intensivo, 60 pazienti in stato di chetoacidosi diabetica. In entrambe le esperienze i pazienti sono stati seguiti fino alla risoluzione dello stato di chetoacidosi e valutati relativamente alla normalizzazione dei parametri clinici caratteristici. Entrambi gli studi hanno verificato che la nuova strategia ha consentito l'ottenimento di outcome clinici sovrapponibili a quelli conseguibili con l'infusione continua di insulina regolare e una significativa riduzione dei costi dipendente dal diverso regime di ricovero (reparto non intensivo vs Terapia Intensiva).

Una esperienza simile a quella citata è stata condotta presso l'Unità Complessa Medicina Interna 2 dell'Ospedale di Sassari verificando, anche nel setting sanitario italiano, risultati favorevoli all'uso dell'analogo dell'insulina Lispro.
* U.O.Medicina Interna 2 Osp.Civ. "SS.Annunziata" ASL1 Sassari 


\begin{tabular}{lcc} 
& Lispro & Controllo \\
\hline Numero & $10 \mathrm{f}$ & $10 \mathrm{f}$ \\
Età media & 63,6 & 74,2 \\
Glicemia basale & $401,6(+/-98,8 \mathrm{ds})$ & $401,6(+/-96,7 \mathrm{ds})$ \\
IDDM e/o trat. insulinico & 8 & 9 \\
NIDDM & 2 & 1 \\
Con patol. infiamm. acute & 3 & 4 \\
Con patol. cardiov. e/o endoc. & 3 & 3 \\
Altre patologie & 4 & 3 \\
\hline
\end{tabular}

Tabella I

Variabili anagrafiche e cliniche dei pazienti nei due gruppi considerati

\section{MATERIALIEMETODI}

Sono stati consecutivamente studiati, durante 4 mesi di osservazione, 20 pazienti ricoverati per diabete mellito chetoacidosico. Di questi, i primi 10 sono stati trattati con l'infusione continua di insulina regolare e i successivi 10 pazienti con l'impiego sottocutaneo dell' analogo dell'insulina Lispro (Tabella I). Il regime terapeutico è descritto in Tabella II. I dati relativi alle variabili anagrafiche e cliniche sono stati analizzati con statistica descrittiva per produrre i valori medi e deviazione standard. Le differenze delle medie, tra i due gruppi, relative alle variabili cliniche di endpoint (valori glicemici, parametri della chetoacidosi e numero di giornate alla risoluzione) sono state valutate con il test $\mathrm{t}$.

La valutazione clinica prevedeva la verifica della normalizzazione dei valori glicemici plasmatici ed il superamento dello stato di chetoacidosi.

L'analisi dei costi è stata fatta assumendo la prospettiva dell'Azienda Ospedaliera e quindi considerando i costi giornalieri reali di ricovero nel Reparto di Medicina che l'Ufficio di Gestione della stessa struttura ha indicato essere pari a 335,70 euro. In quelle strutture in cui si ricorre regolarmente, per la risoluzione di questi casi, al Reparto di Terapia Intensiva, i costi di gestione del trattamento intensivo con insulina sono sicuramente superiori. Un recente lavoro di Lucioni ha stimato in 1.033,43 euro

\begin{tabular}{lcc}
\hline & $\begin{array}{c}\text { Insulina } \\
\text { Lispro }\end{array}$ & $\begin{array}{c}\text { Insulina } \\
\text { Regolare }\end{array}$ \\
\hline $\begin{array}{l}\text { Dose } \\
\text { Via di somministrazione }\end{array}$ & $\begin{array}{c}5 \mathrm{u} . / \mathrm{h} . \\
\text { sottocutanea }\end{array}$ & $\begin{array}{c}5 \mathrm{u} . / \mathrm{h} . \\
\text { infusione continua }\end{array}$ \\
\hline
\end{tabular}

Tabella II

Regimi terapeutici messi a confronto nello studio il costo medio di un giorno di ricovero in terapia intensiva contro i 299,54 euro di un reparto non intensivo [11].

La riduzione dei costi con una delle due strategie a confronto è stata computata come riduzione del numero di giornate di ricovero per ottenere la risoluzione del quadro di scompenso metabolico.

\section{RISULTATI}

I risultati clinici ottenuti con le procedure sopra descritte sono riassunti in Tabella III.

I gruppi a confronto sono risultati confrontabili per storia clinica e valori glicemici al baseline (Tabella I).

Entrambi i trattamenti hanno consentito il raggiungimento di una normalizzazione del compenso metabolico, con differenze statisticamente non significative per quanto riguarda la concentrazione dei chetoni urinari, l'acidità plasmatica, l'assunzione quotidiana di liquidi e il numero giornaliero di unità di insulina. Una differenza importante è stata invece rilevata per quanto riguarda i tempi di raggiungimento della normalizzazione nei due gruppi a confronto, con una media di 4,8 giornate nel gruppo di trattamento con l'analogo dell'insulina Lispro verso 6,90 giornate nel gruppo trattato con l'infusione di insulina regolare, comprendendo un periodo di osservazione successivo alla normalizzazione di qualche ora (12-24), omogeneo nei due gruppi. Tale dato è più che sufficiente a determinare differenze importanti nei costi di degenza che raggiungono, per ogni paziente, 704,97 euro per ricovero. Oltre a ciò, si è assistito anche ad una riduzione dei tempi di assistenza infermieristica al letto del paziente per la somministrazione della terapia insulinica a causa delle diverse caratteristiche farmacologiche dei due preparati. Ne consegue, quindi, che attraverso una valutazione dei parametri di spesa che non preveda il semplice computo dei costi giornalieri totali di reparto ottenuti dividendo la spesa annua totale per il numero di giornate "ricovero" prodotte, si potrebbe evidenziare, oltre alla riduzione del numero delle giornate stesse, anche una ottimizzazione dei tempi di assistenza intra-reparto.

Considerando la media dei ricoveri per anno nel reparto di Medicina e l'incidenza su questi dei ricoveri di pazienti con scompenso acuto si può stimare il numero totale di giornate di degenza risparmiate per anno e computare la relativa riduzione di costi annuale (Tabella IV).

\section{DISCUSSIONE}

L'esperienza condotta presso l'U.O. di Medicina Interna 2 dell'Azienda Ospedaliera di Sassari ha confermato i risultati di riduzione 


\begin{tabular}{llll}
\hline & \multicolumn{1}{c}{ Lispro } & \multicolumn{1}{c}{ Controllo } \\
\hline Chet. urinari 24h (mg/dl) & $7(+/-6,3)$ & $14(+/-10,22)$ & T - test $(\mathrm{P}) 0,082$ \\
Osmol. pl 24h (mOsm/Kg) & $306,1(+/-6,67)$ & $315,9(+/-16,7)$ & $\mathrm{ns}$ \\
Normalizzazione (gg.) & $4,80(+/-1,6)$ & $6,90(+/-2,6)$ & $\mathrm{P}=0,044^{\star *}$ \\
Liquidi/die (ml) & $950(+/-282,2)$ & $1.200(+/-258,2)$ & 0,054 \\
Glicemia media (mg/dl) & $157,1(+/-41,7)$ & $170,6(+/-29,9)$ & $\mathrm{ns}$ \\
Un.tot.insulina (U.) & $26(+/-9)$ & $28,1(+/-5,9)$ & $\mathrm{ns}$ \\
\hline
\end{tabular}

Tabella III Parametri clinici laboratoristici dei costi di assistenza già evidenziati dalle esperienze internazionali $[9,10]$ con l'impiego dell' analogo dell'insulina Lispro in luogo dell'insulina umana regolare per il trattamento dello scompenso acuto diabetico. Il risultato descritto è stato raggiunto attraverso una riduzione del numero di giornate di ricovero, ma si sarebbe potuto evidenziare anche un abbassamento dell'intensità di assistenza del personale medico e infermieristico al letto del paziente.

I limiti del presente lavoro sono identificabili soprattutto nel basso numero di soggetti studiati in conseguenza del breve tempo di osservazione. Tuttavia, considerando che i nostri Reparti di medicina assistono annualmente circa 66 pazienti con chetoacidosi diabetica, possiamo affermare che il campione è sufficientemente rappresentativo di questa realtà.

La positività della nostra esperienza suggerisce, in un futuro prossimo, la necessità di un allargamento della casistica osservata in modo da rendere più rappresentativi i risultati. In questa circostanza si cercherà di operare una più attenta valutazione dei costi, rintracciabili non solo nel numero di giornate di degenza ma anche nel diverso impiego del personale infermieristico.

\subsection{0}

3,3\% (ca.66 pazienti)

335,70 euro $46.481,12$ euro

\section{Tabella IV}

Valutazione di costi e risparmi

\section{BIBLIOGRAFIA}

1. Basu A, Close CF, Jenkins D, Krentz AJ, Nattrass M, Wright AD. Persisting mortality in diabetic ketoacidosis. Diabet Med 1993; 10:282-284,

2. White NH. Diabetic ketoacidosis in children. Endocrinol Metab Clin North Am 2000; 29:657-682,

3. Kitabchi AE, Umpierrez GE, Murphy MB, Barrett EJ, Kreisberg RA, Malone JI, Wall BM. Management of hyperglycemic crises in patients with diabetes. Diabetes Care 2001;24:131-153

4. Alberti KG. Low-dose insulin in the treatment of diabetic ketoacidosis. Arch Intern Med 1977; 137:1367-1376

5. Lebovitz HE. Diabetic ketoacidosis. Lancet 1995; 345:767-772

6. Fisher JN, Shahshahani MN, Kitabchi AE. Diabetic ketoacidosis: low-dose insulin therapy by various routes. N Engl J Med 1977; 297:238-241 
7. Alberti KG, Hockaday TD, Turner RC. Small doses of intramuscular insulin in the treatment of diabetic "coma". Lancet 1973; 2:515-522

8. Kitabchi AE, Ayyagari V, Guerra SM. The efficacy of low-dose versus conventional therapy of insulin for treatment of diabetic ketoacidosis. Ann Intern Med 1976; 84:633-638

9. Della Manna T, Steinmetz L, Campos PR et al. Subcutaneous use of a fast-acting insulin analog: an alternative treatment for pediatric patients with diabetic ketoacidosis. Diabetes Care. 2005; 28(8):1856-61

10. Umpierrez G, Latif K, Stoever J, Cuervo R et al. Efficacy of subcutaneous insulin lispro versus continuous intravenous regular insulin for the treatment of patients with diabetic ketoacidosis. American Journal of Medicine, 2004, 117: 291-296 e 357-358

11. Lucioni C et al. I costi della sepsi in Italia. Farmeconomia e percorsi terapeutici, 2001, 2(3): 19-28 